\title{
Remarkable findings of mosses from the Orthotrichaceae family in the Muránska planina National Park (Slovakia)
}

\author{
Vítězslav Plášek, Drahoš Blanár, Lucie Fialová \& Zuzana Skoupá
}

Remarkable findings of mosses from Orthotrichaceae family in the Muránska planina National Park (Slovakia). - Acta Mus. Siles. Sci. Natur. 65: 167-178, 2016.

\begin{abstract}
The article presents recent findings of epiphytic bryophytes from the Orthotrichaceae family in the Muránska planina National Park. Field surveys were carried out between 2008 and 2016. In total, 111 localities were visited. Altogether, 19 taxa from the Orthotrichaceae family were recorded - 8 of them are new for the territory of the National Park (Dorcadion affine var. bohemicum, D. rupestre, Orthotrichum diaphanum, O. patens, O. scanicum, O. stramineum, Pulvigera lyellii, and Ulota bruchii) and 1 as new for Slovakia (Dorcadion affine var. bohemicum). Eleven species are listed on the Red list of bryophytes of Slovakia - EX: Orthotrichum rogeri; CR: Nyholmiella gymnostoma, Orthotrichum patens, O. scanicum; VU: Dorcadion striatum, Ulota bruchii; NT: Dorcadion speciosum, Orthotrichum pallens, Pulvigera lyellii, Ulota crispa; DD: Orthotrichum stramineum. The most interesting findings are briefly discussed.
\end{abstract}

Key words: Epiphytic bryophytes, Dorcadion, Nyholmiella, Orthotrichum, Pulvigera, Ulota, endangered taxa

\section{Introduction}

Muránska planina National Park is situated in the western part of the Slovenské rudohorie Mts in the West Carpathians. In the protected area there are two types of relief: karst, found on Triassic limestones of the orographic unit of the Muránska planina Mts; and non-karst, resting on crystallic slates, granitoids and Neogene sediments that in general belong to the orographic units of the Veporské vrchy Mts and Stolické vrchy Mts. Volcanites occur very rarely in the karst area. The karst relief forms the central part of the National Park with well-preserved karst plain and karst processes found in some places, both on the surface and underground (cf. Mitter 1991).

Owing to its location near to the border of the Carpathians and the Pannonian Basin, the territory of Muránska planina National park is a very valuable natural region. Its major part embraces a karst landscape with extraordinary natural habitat and ecosystem diversity hosting many plant species. This diversity is mostly caused by variable geological and geomorphological conditions as well as by the position near to the southern margin of the Western Carpathians, which is warmer and drier than the central part of this mountain range (Kochjarová et al. 2015). Due to the high number and unusual diversity of plant species and their communities the Muránska planina Mts represent one of the most interesting and most valuable natural science areas, not only in Slovakia but also in the whole Carpathians (Blanár \& Kochjarová 2012).

Approximately $90 \%$ of the territory of Muránska planina National Park is covered by forests, dominated by beech and fir-beech which, along with subalpine beech forests, are spread mainly on the plateau and also in the northern part of the Muránska planina National Park.

According to Šoltés et al. (2004), 372 taxa of bryophytes (98 liverworts and 274 mosses), representing about $40 \%$ of the bryoflora of Slovakia, have been recorded in the area of the Muránska planina National Park and in the adjacent area (including mainly its buffer zone).

\section{Material and Methods}

Specimens were collected in the Muránska planina National Park and its buffer zone during 2008-2015. The research sites were selected with suitable ecological conditions for the growth of epiphytes, mainly on the edges of deciduous and mixed forests, alleyways along roads, old orchards and parks, solitary trees, willows, wetlands and sites located along streams. Sites were selected in order to cover the largest portion of the studied area. All the herbarium specimens are hou- 
sed in OSTR. Most of the recent material was collected by Lucie Fialová (in the course of her diploma thesis), the remainder being collected by other authors. Identifications of all specimens were verified by Vítězslav Plášek. A map of studied localities in the National Park Muránska planina was made with using QGIS software (Map 1).

The nomenclature of the moss taxa follows that of Plášek et al. (2015), where a new taxonomical arrangement of the traditionally conceived genera Orthotrichum and Ulota was presented. Threatened categories (placed in square brackets after the names of taxa) follow Red List of Slovakian bryophytes by Kubinská et al. 2001.

\section{A LIST OF RECENTLY VISITED LOCALITIES}

Data of the localites are presented in the following order: Orographical unit; village/town (= cadastre); locality, site location, altitude, GPS coordinates in WGS 84 (latitude, longitude; with an accuracy of $\pm 5-10$ $\mathrm{m})$.

1 Veporské vrchy Mts, Polomka, $1.2 \mathrm{~km}$ W of Polomka village, $1.8 \mathrm{~km}$ SE of Bacúch village, Petríkovo valley (near a public road), $580 \mathrm{~m}$ a.s.1., GPS = N48 50'53.000", E1950'04.400".

2 Muránska planina Mts, Pohorelá, intravilan of Pohorelská Maša village, in the park, $695 \mathrm{~m}$ a.s.1., GPS = N48 50 '38.832", E2002'10.644".

3 Muránska planina Mts, Valkovňa; $450 \mathrm{~m} \mathrm{~W}$ of Nová Maša village, $1.2 \mathrm{~km}$ SE of Pohorelská Maša village, $730 \mathrm{~m}$ a.s.1., GPS = N48 ${ }^{\circ}$ 0'05.100", E2002'35.221".

4 Veporské vrchy Mts, Závadka nad Hronom, $1.5 \mathrm{~km}$ S of Závadka nad Hronom village, valley NE of Kochlovec hill, 705 m a.s.1., GPS = N48 50'00.733", E=1955'00.759".

5 Muránska planina Mts.; Šumiac; $1.4 \mathrm{~km}$ E of Zlatno village, $2.1 \mathrm{~km}$ NW of Červena Skala village, near a public road, 780 m a.s.1., GPS = N4849'45.408", E2006'23.148".

6 Muránska planina Mts, Valkovňa, 1.1 km SW of Nová Maša village, 2 km SE of Pohorelská Maša village, 735 m a.s.1., GPS = N4849'39.000", E2002'17.041".

7 Muránska planina Mts, Valkovňa, $250 \mathrm{~m} \mathrm{~S}$ of Zlatno village, $1.4 \mathrm{~km}$ SE of Valkovňa village, $760 \mathrm{~m}$ a.s.1., GPS = N4849'33.841", E2005'14.280"

8 Veporské vrchy Mts, Polomka, $2.5 \mathrm{~km} \mathrm{~S}$ of Polomka village, $5 \mathrm{~km}$ SW of Závadka nad Hronom village, Petríkovo valley, 760 m a.s.1., GPS = N4849'29.900", E1951'00.800".

9 Muránska planina Mts, Valkovňa, $1.6 \mathrm{~km} \mathrm{SW}$ of Nová Maša village, $2.5 \mathrm{~km}$ SE of Pohorelská Maša village, $745 \mathrm{~m}$ a.s.1., GPS = N4849'20.759", E2002'14.399".

10 Muránska planina Mts, Šumiac, $1.7 \mathrm{~km} \mathrm{SW}$ of Zlatno village, $1.9 \mathrm{~km}$ SE of Nová Maša village, Havraník mixed mire, 750 m a.s.1., GPS = N48 49'06.060", E2003'46.260".

11 Veporské vrchy Mts, Závadka nad Hronom, $3.4 \mathrm{~km} \mathrm{~S}$ of Závadka nad Hronom village, $798 \mathrm{~m}$ a.s.1., GPS = N4849'00.169", E1956'00.207".

12 Muránska planina Mts, Šumiac, $800 \mathrm{~m}$ SW of Červená Skala village, 3.9 km SE of Zlatno village, Trsteník valley, near a stream, $800 \mathrm{~m}$ a.s.1., GPS = N4848'45.972", E2007'56.316".

13 Veporské vrchy Mts, Závadka nad Hronom, $5.2 \mathrm{~km} \mathrm{~S}$ of Závadka nad Hronom village, $853 \mathrm{~m}$ a.s.1., GPS = N4848'00.505", E1956'00.298".

14 Muránska planina Mts.; Šumiac; $1.5 \mathrm{~km} \mathrm{SW}$ of Červená Skala village, Trsteník valley, $814 \mathrm{~m}$ a.s.l., GPS = N48 48'00.224", E2007'00.451".

15 Muránska planina Mts, Závadka nad Hronom, Hronec valley, $2.3 \mathrm{~km} \mathrm{NW}$ of Kl'ak Mt., loc. 'Za Nihovom', $810 \mathrm{~m}$ a.s.1., GPS = N4847'46.979", E1956'55.860".

16 Muránska planina Mts, Závadka nad Hronom, Hronec valley, $6.5 \mathrm{~km} \mathrm{~S}$ of Závadka village, $2.7 \mathrm{~km} \mathrm{NW}$ of Kl'ak Mt., 795 m a.s.l., GPS = N4847'40.668" 1956'12.664".

17 Muránska planina Mts, Závadka nad Hronom, $1.8 \mathrm{~km}$ NW of Kl'ak Mt., $8.2 \mathrm{~km}$ SW of Nová Maša village, N slope of 'Vel'ká Stožka' NNR, 830 m a.s.1., GPS = N4847'36.800", E1957'15.000".

18 Muránska planina Mts, Závadka nad Hronom, Hronec valley, $1.7 \mathrm{~km} \mathrm{NW}$ of Kl'ak Mt., loc. 'Za Nihovo', $840 \mathrm{~m}$ a.s.1., GPS = N4847'36.179", E1957'24.779"

19 Muránska planina Mts, Závadka nad Hronom, $1.8 \mathrm{~km} \mathrm{NW}$ of Kl'ak Mt., $8.2 \mathrm{~km}$ SW of Nová Maša village, loc. 'Vel'ká Stožka' NNR, 850 m a.s.1., GPS = N4847'34.200", E1957'22.200".

20 Muránska planina Mts, Závadka nad Hronom, Hronec valley, $1.4 \mathrm{~km} \mathrm{NW}$ of Kl'ak Mt., loc. 'Za Nihovom', $850 \mathrm{~m}$ a.s.1., GPS = N48 47'31.380", E1957'49.02".

21 Muránska planina Mts, Hel'pa, $2.5 \mathrm{~km} \mathrm{SW}$ of Polomka village, $5 \mathrm{~km}$ SW of Závadka nad Hronom village, part 'Za Nihovo', 740 m a.s.1., GPS = N4847'25.944", E1959'02.112".

22 Muránska planina Mts, Muránska Huta, $1.3 \mathrm{~km}$ NE of Muránska Huta village, $1.2 \mathrm{~km}$ SE of 'Javorinka' mountain saddle, loc. 'Tesná Skala' rock, 895 m a.s.1., GPS = N4847'12.696", E2007'31.044".

23 Muránska planina Mts; Muráň; 6 km NW of Muráň village, 1.8 km NE of Kl'ak Mt., loc. 'Vel'ká Stožka' NNR, 1250 m a.s.1., GPS = N4847'11.904", E1959'19.428" 
24 Muránska planina Mts, Muránska Huta, $1.4 \mathrm{~km}$ NE of Muránska Huta village, $3.4 \mathrm{~km} \mathrm{~S}$ of Červená Skala village, loc. 'Tesná Skala' rock, $870 \mathrm{~m}$ a.s.1., GPS = N48 47'10.716", E2007'30.936".

25 Muránska planina Mts, Muráň, $7.5 \mathrm{~km} \mathrm{~S}$ of Závadka nad Hronom village, valley of Hronec river, loc. 'Klatná', 827 m a.s.l., GPS $=\mathrm{N} 48^{\circ} 47^{\prime} 00.961 ", \mathrm{E} 19^{\circ} 56^{\prime} 00.381^{\prime \prime}$.

26 Muránska planina Mts, Muráň, 7 km NW of Muránská Huta village, NW of Vel'ký Cigáñ Mt., 1134 m a.s.l., GPS = N484'00.495", E2001'00.367".

27 Muránska planina Mts, Muráň, $8 \mathrm{~km} \mathrm{NW}$ of Muránská Huta village, loc. 'Studňa', $1210 \mathrm{~m}$ a.s.l., GPS = N48 $47^{\prime} 00.332 "$, E $20^{\circ} 00^{\prime} 00.344^{\prime \prime}$.

28 Muránska planina Mts, Muráň, 7.5 km NW of Muránská Huta village, W slope of Vel'ký Cigáň Mt., 1202 m a.s.l., GPS $=$ N48 $47^{\circ} 00.290^{\prime \prime}, \mathrm{E} 20^{\circ} 00^{\prime} 00.530^{\prime \prime}$.

29 Muránska planina Mts, Muráň, $9.3 \mathrm{~km}$ NW of Muránská Huta village, SW slope of Sitárka Mt., $1087 \mathrm{~m}$ a.s.l., GPS $=\mathrm{N} 48^{\circ} 47^{\prime} 00.166^{\prime \prime}, \mathrm{E} 19^{\circ} 59^{\prime} 00.028^{\prime \prime}$.

30 Muránska planina Mts, Závadka nad Hronom, $8 \mathrm{~km}$ SE of Závadka nad Hronom village, $800 \mathrm{~m}$ E of Klak Mt., loc. 'Vel'ká Stožka' NNR, 1260 m a.s.1., GPS = N4846'69.800", E1957'21.204".

31 Muránska planina Mts, Muráň, 8.5 km NW of Muránská Huta village, SE slope of Sitárka Mt., 1204 m a.s.l., GPS $=\mathrm{N} 48^{\circ} 46^{\prime} 49.036^{\prime \prime}, \mathrm{E} 19^{\circ} 59^{\prime} 15.069^{\prime \prime}$.

32 Muránska planina Mts, Muráň, $2.3 \mathrm{~km}$ W of Kl'ak Mt., $3.8 \mathrm{~km}$ E of Fabova hol’a Mt., N slope of loc. 'Vel'ká Stožka' NNR, 780 m a.s.l., GPS $=$ N48 $46^{\prime} 42.528^{\prime \prime}$, E19 $10^{\circ}$ '07.980".

33 Muránska planina Mts, Muráň, 3.6 km SW of Kl'ak Mt., 2.4 km E of Fabova hol’a Mt., loc. 'Malá Stožka' NNR, 930 m a.s.l., GPS $=\mathrm{N} 48^{\circ} 46^{\prime} 32.808^{\prime \prime}, \mathrm{E} 19^{\circ} 55^{\prime} 03.900^{\prime \prime}$.

34 Muránska planina Mts, Muráń, $8.6 \mathrm{~km} \mathrm{~S}$ of Závadka nad Hronom village, $8.5 \mathrm{~km} \mathrm{NW}$ of Muráň village, loc. 'Malá Stožka' NNR, 960 m a.s.l., GPS = N4846'15.800", E1955'55.000".

35 Muránska planina Mts, Muráň, $4.3 \mathrm{~km} \mathrm{NE}$ of Muráň village, $1.5 \mathrm{~km} \mathrm{~W}$ of Predná Hora hill, loc. 'Bielé vody', $650 \mathrm{~m}$ a.s.l., GPS = N48 $46^{\prime} 12.000^{\prime \prime}, \mathrm{E} 20^{\circ} 05^{\prime} 22.800^{\prime \prime}$.

36 Muránska planina Mts, Muráń, $3.9 \mathrm{~km} \mathrm{NE}$ of Muráň village, $1.8 \mathrm{~km} \mathrm{NW}$ of Predná Hora hill, loc. 'Šiance' , $605 \mathrm{~m}$ a.s.l., GPS = N48 $46^{\prime} 01.193^{\prime \prime}, \mathrm{E} 20^{\circ} 04^{\prime} 50.790^{\prime \prime}$.

37 Muránska planina Mts, Muráň, $10 \mathrm{~km} \mathrm{NW}$ of Muráňská Huta village, E slope of Mt. Klak, $1316 \mathrm{~m}$ a.s.l., GPS = N484ㅇ' $00.808^{\prime \prime}, \mathrm{E} 19^{\circ} 58^{\prime} 00.323^{\prime \prime}$.

38 Stolické vrchy Mts, Murán̆, $800 \mathrm{~m}$ SW of Muránska Huta, W foot of Predná Hora hill, $759 \mathrm{~m}$ a.s.l., GPS = N4846'00.341", E2006'00.259".

39 Stolické vrchy Mts, Muráň, 4 km NE of Muráň village, loc. Predná Hora, SW of the Predná Hora Castle, $830 \mathrm{~m}$ a.s.l., GPS = N48 $46^{\prime} 00.300^{\prime \prime}, \mathrm{E} 20^{\circ} 05^{\prime} 17.700^{\prime \prime}$.

40 Muránska planina Mts, Murán̆, $3.3 \mathrm{~km} \mathrm{NE}$ of Muráň village, $2 \mathrm{~km} \mathrm{SW}$ of Predná Hora hill, loc. 'Biele vody', $530 \mathrm{~m}$ a.s.l., GPS = N48 $45^{\prime} 54.781^{\prime \prime}, \mathrm{E} 20^{\circ} 04^{\prime} 55.981^{\prime \prime}$.

41 Muránska planina Mts, Muráń, $3.4 \mathrm{~km} \mathrm{NE}$ of Muráň village, $2 \mathrm{~km} \mathrm{SW}$ of Predná Hora hill, loc. 'Šiance' NNR, 540 m a.s.l., GPS $=\mathrm{N} 48^{\circ} 45^{\prime} 56.639^{\prime \prime}, \mathrm{E} 20^{\circ} 04^{\prime} 57.119^{\prime \prime}$.

42 Stolické vrchy Mts, Murán̆, $3.8 \mathrm{~km} \mathrm{NW}$ of Muránska Zdychava village, $1.5 \mathrm{~km} \mathrm{SW}$ of Muránska Huta village, Predná Hora hill, $790 \mathrm{~m}$ a.s.1., GPS = N4845'46.944", E2006'15.012".

43 Muránska planina Mts, Muráň, $2.1 \mathrm{~km} \mathrm{NE}$ of Murán̆ village, $3.6 \mathrm{~km} \mathrm{SW}$ of Predná Hora hill, near a path to the 'Muránský hrad' ruin, $860 \mathrm{~m}$ a.s.l., GPS = N48 $45^{\prime} 41.600^{\prime}$, E20 $03^{\circ} 40.600^{\prime \prime}$.

44 Muránska planina Mts, Murán̆, $8.9 \mathrm{~km} \mathrm{NE}$ of Tisovec town, $4.7 \mathrm{~km}$ NW of Muráň village, loc. 'Hrdzavá' NNR, 940 m a.s.l., GPS $=$ N48 $45^{\prime} 34.524 "$, E19 $58^{\prime} 45.048^{\prime \prime}$.

45 Muránska planina Mts, Muráň, $3.7 \mathrm{~km} \mathrm{SW}$ of Muránska Huta village, $2.3 \mathrm{~km} \mathrm{NE}$ of Muráň village, loc. 'Šiance' NNR, 430 m a.s.l., GPS $=$ N48 $45^{\prime} 23.700^{\prime \prime}$, E20 $04^{\circ} 19.600^{\prime \prime}$.

46 Muránska planina Mts, Muráň, 3.8 km SW of Muránská Huta village, S of loc. 'Vel'ká Lúka', 854 m a.s.l., GPS = $\mathrm{N} 48^{\circ} 45^{\prime} 00.900^{\prime \prime}, \mathrm{E} 20^{\circ} 03^{\prime} 00.796^{\prime \prime}$.

47 Muránska planina Mts, Muráň, $5.5 \mathrm{~km} \mathrm{NW}$ of Muráň village, upper part of Hrdzavá dolina valley, $969 \mathrm{~m}$ a.s.l., GPS $=\mathrm{N} 48^{\circ} 45^{\prime} 00.823 ", \mathrm{E} 19^{\circ} 58^{\prime} 00.434^{\prime \prime}$.

48 Muránska planina Mts, Muráń, $1.5 \mathrm{~km}$ NE of Muráň village, SE edge of Cigánka hill, $416 \mathrm{~m}$ a.s.l., GPS = N48 $45^{\prime} 00.424 "$, E200 $04^{\prime} 00.174 "$.

49 Muránska planina Mts, Muráň, $5 \mathrm{~km} \mathrm{SW}$ of Muráňská Huta village, 0.7 km NE of 'Muránský Hrad' ruin, $658 \mathrm{~m}$ a.s.l., GPS = N48 $45^{\prime} 00.271^{\prime \prime}$, E20 $02^{\prime} 00.982^{\prime \prime}$.

50 Muránska planina Mts, Murán̆, 4 km NW of Muráň village, middle part of Hrdzavá dolina valley, $833 \mathrm{~m}$ a.s.l., GPS $=\mathrm{N} 48^{\circ} 45^{\prime} 00.117^{\prime \prime}, \mathrm{E} 19^{\circ} 59^{\prime} 00.457^{\prime \prime}$.

51 Muránska planina Mts, Muráň, $1.4 \mathrm{~km}$ NE of Muráň village, loc. 'Cigánka' NNR, $674 \mathrm{~m}$ a.s.l., GPS = $\mathrm{N} 48^{\circ} 45^{\prime} 00.103^{\prime \prime}, \mathrm{E} 20^{\circ} 02^{\prime} 00.935^{\prime \prime}$.

52 Muránska planina Mts, Muráň, $1.5 \mathrm{~km} \mathrm{~N}$ of Muráň village, $491 \mathrm{~m}$ a.s.l., GPS = N48 45'00.069", E20 $02^{\prime} 00.211^{\prime \prime}$.

53 Muránska planina Mts, Muráň, $4.5 \mathrm{~km} \mathrm{NW}$ of Muráň village, upper part of Hrdzavá valley, $836 \mathrm{~m}$ a.s.l., GPS = N484'ㄷ․019", E1958'00.065".

54 Muránska planina Mts, Muráň, 3.1 km NW of Muráň village, $4.4 \mathrm{~km} \mathrm{NW}$ of Muránska Lehota village, loc. 'Hrdzavá' NNR, 675 m a.s.l., GPS = N4844'57.732", E1959'49.236". 


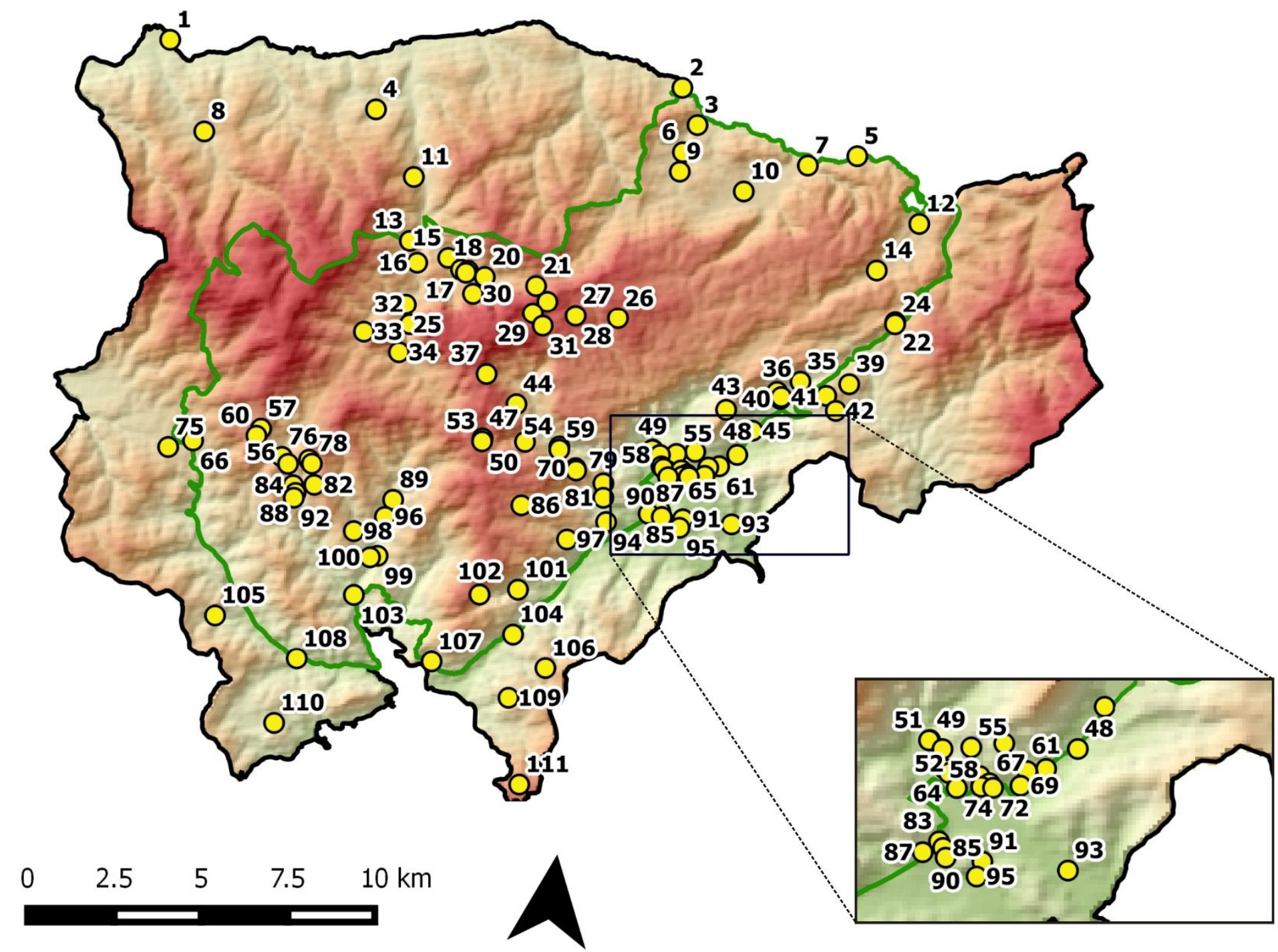

Map 1: Map of study area with visited localities - border of Muránska planina National Park indicated by green line; border of the buffer zone indicated by black line. 
55 Muránska planina Mts, Muráň, $800 \mathrm{~m} \mathrm{~N}$ of Muráň village, $5.3 \mathrm{~km} \mathrm{SW}$ of Predná Hora hill, 580 m a.s.1., GPS $=\mathrm{N} 48^{\circ} 44^{\prime} 57.700^{\prime \prime}, \mathrm{E} 20^{\circ} 02^{\prime} 34.500^{\prime \prime}$.

56 Veporské vrchy Mts, Tisovec, $5.7 \mathrm{~km}$ E of Pohronská Polhora village, $2.2 \mathrm{~km} \mathrm{NE}$ of 'Zbojská' mountain pass, loc. 'Kučelach', 1050 m a.s.1., GPS = N4844'56.600", E1952'47.900".

57 Veporské vrchy Mts, Tisovec, 2.7 km S of Fabova hol'a Mt., 7.2 km SW of Kl'ak Mt., loc. 'Kučelach', 1040 m a.s.1., GPS = N48 44'56.600", E1952'47.900".

58 Muránska planina Mts, Muráň, $0.7 \mathrm{~km} \mathrm{~N}$ of Muráň village, edge of the village, $516 \mathrm{~m}$ a.s.l., GPS = N4844'55.900", E2002'11.500".

59 Muránska planina Mts, Muráň, $4.3 \mathrm{~km} \mathrm{NW}$ of Muránska Lehota village, $3.1 \mathrm{~km}$ NW of Muráň village, Hrdzavá dolina valley, 710 m a.s.1., GPS = N4844'54.888", E1959'49.416".

60 Veporské vrchy Mts, Tisovec, $5.5 \mathrm{~km}$ SE of Pohronská Polhora village, $2 \mathrm{~km}$ NE of 'Zbojská' mountain pass, loc. 'Kučelach', 1070 m a.s.1., GPS = N4844'49.812", E1952'42.060".

61 Muránska planina Mts, Muráň, $700 \mathrm{~m}$ NE of Muráň village, meadows along public road towards Muránska Huta, 400 m a.s.1., GPS = N4844'48.800", E2003'35.700".

62 Muránska planina Mts, Muráň, $500 \mathrm{~m}$ NE of Muráň village, a quarry near public road towards Muránska Huta, 430 m a.s.1., GPS = N4844'47.000", E2003'20.600".

63 Muránska planina Mts, Muráň, $350 \mathrm{~m} \mathrm{~N}$ of Muráň village, $230 \mathrm{~m}$ E of 'Hrdzavá' NNR, $478 \mathrm{~m}$ a.s.l., GPS = N48 44'45.300", E2002'14.600".

64 Muránska planina Mts, Muráň, 0,5 km NNW of Muráň village, 1,9 km SW of Cigánka hill, 485 m a.s.1., GPS $=\mathrm{N} 48^{\circ} 44^{\prime} 43.400^{\prime \prime}, \mathrm{E} 20^{\circ} 02^{\prime} 17.700^{\prime \prime}$.

65 Muránska planina Mts, Muráň, $350 \mathrm{~m} \mathrm{~N}$ of Muráň village, $5.4 \mathrm{~km}$ SW of Predná Hora hill, along a path towards 'Muránský hrad' ruin, $460 \mathrm{~m}$ a.s.1., GPS = N4844'42.800", E2002'42.700".

66 Veporské vrchy Mts, Tisovec, 3.7 km E of Pohronská Polhora village, $1 \mathrm{~km}$ W of loc. 'Kučelach', loc. 'Zbojská' mountain pass, 746 m a.s.1., GPS = N4844'41.554", E1951'13.453".

67 Muránska planina Mts, Muráň, $250 \mathrm{~m} \mathrm{~N}$ of Murán̆ village, a quarry near cemetery, $435 \mathrm{~m}$ a.s.l., GPS = N4844'39.300", E2002'51.036".

68 Muránska planina Mts, Muráň, $200 \mathrm{~m}$ NW of Muráň village, near a public road towards Muránska Huta, 400 m a.s.1., GPS $=\mathrm{N} 48^{\circ} 44^{\prime} 39.000^{\prime \prime}, \mathrm{E} 20^{\circ} 03^{\prime} 16.000^{\prime \prime}$.

69 Muránska planina Mts, Muráň, $1.8 \mathrm{~km} \mathrm{NE}$ of Muránska Lehota village, edge of Murán̆ village, along a public road, 400 m a.s.1., GPS = N48 44'39.000", E2003'16.000".

70 Muránska planina Mts, Muráň, $3.6 \mathrm{~km}$ NW of Muráňska Lehota village, $2.5 \mathrm{~km}$ NW of Murán̆ village, loc. 'Vrbjarka', 970 m a.s.1., GPS = N48 44'38.580", E2000'13.896".

71 Muránska planina Mts, Muráň, $200 \mathrm{~m} \mathrm{~N}$ of Muráň village, $5.4 \mathrm{~km} \mathrm{SW}$ of Predná Hora hill, along a path to the 'Muránský hrad' ruin, 430 m a.s.1., GPS = N4844'37.200", E2002'44.500".

72 Muránska planina Mts, Muráň, Muráň village, near a cemetery, $420 \mathrm{~m}$ a.s.1., GPS = N48 44'37.000", E2002'54.000".

73 Muránska planina Mts, Muráň, $3.6 \mathrm{~km}$ NW of Muránska Lehota village, $2.5 \mathrm{~km}$ NW of Muráň village, loc. 'Mátožná', 980 m a.s.1., GPS = N4844'36.528", E2000'14.940".

74 Muránska planina Mts, Muráň, Muráň village, $100 \mathrm{~m} \mathrm{SW}$ of a cemetery, $420 \mathrm{~m}$ a.s.1., GPS = N48 44'35.700", E2002'24.900".

75 Veporské vrchy Mts, Pohronská Polhora, $3 \mathrm{~km} \mathrm{SE}$ of Pohronská Polhora village, $0.9 \mathrm{~km} \mathrm{~S}$ of Zbojska hill, loc. 'Borovniak' quarry, 730 m a.s.1., GPS = N4844'33.972", E1950'38.958".

76 Veporské vrchy Mts, Tisovec, 3.7 km NE of Bánovo settlement, 3 km N of Magnet hill, Strieborná hill, 790 $\mathrm{m}$ a.s.1., GPS = N48 44'31.700", E1953'20.000".

77 Veporské vrchy Mts, Tisovec, $7.2 \mathrm{~km} \mathrm{NW}$ of Tisovec town, $3 \mathrm{~km}$ NE of Magnet hill, loc. 'Tisovec-Roveň', 935 m a.s.1., GPS $=$ N48 $44^{\prime} 30.660^{\prime \prime}$, E19 $13^{\circ} 3^{\prime} 58.272^{\prime \prime}$.

78 Veporské vrchy Mts, Tisovec, 3.7 km SE of Fabova hol'a Mt., 6.4 km SW of Kl'ak Mt., near loc. 'TisovecRoveň', 905 m a.s.1., GPS = N4844'27.168", E1954'02.592".

79 Muránska planina Mts, Muráň, $2.8 \mathrm{~km}$ NW of Muránska Lehota village, $1.7 \mathrm{~km}$ W of Muráň village, near loc. 'Mátožná', 750 m a.s.1., GPS = N4844'26.160", E2000'54.396".

80 Veporské vrchy Mts, Tisovec, $3.6 \mathrm{~km} \mathrm{NE}$ of Bánovo settlement, $2.8 \mathrm{~km} \mathrm{~N}$ of Magnet hill, slope of Strieborná hill, 760 m a.s.1., GPS = N4844'25.600", E1953'29.100".

81 Muránska planina Mts, Muráň, $2.5 \mathrm{~km}$ NW of Muráňska Lehota village, $1.7 \mathrm{~km}$ W of Muráň village, 'Kubíčková dolina' valley, loc. 'Javorníková' NNR, 570 m a.s.1., GPS = N4844'12.804", E2000'55.584".

82 Veporské vrchy Mts, Tisovec, $4.3 \mathrm{~km}$ SE of Fabova hol'a Mt., $2.5 \mathrm{~km}$ NE of Magnet hill, near loc. 'TisovecRoveň', 930 m a.s.1., GPS = N4844'07.692", E1954'07.848".

83 Muránska planina Mts, Muráň, 1.1 km NW of Muránska Lehota village, $400 \mathrm{~m}$ SW of Murán̆ village, $440 \mathrm{~m}$ a.s.1., GPS = N48 44'06.791", E2002'13.319".

84 Veporské vrchy Mts, Tisovec, $3.4 \mathrm{~km} \mathrm{NE}$ of Bánovo settlement, $2.2 \mathrm{~km} \mathrm{NE}$ of Magnet hill, slope of Strieborná hill, $750 \mathrm{~m}$ a.s.1., GPS = N4844'06.100", E1953'38.300". 
85 Stolické vrchy Mts, Muráň, $1.1 \mathrm{~km}$ NW of Muránská Lehota village, $650 \mathrm{~m}$ SW of Muráň village, $435 \mathrm{~m}$ a.s.1., GPS = N48 $44^{\prime} 03.500^{\prime \prime}$, E2002'16.900".

86 Muránska planina Mts, Muráň, 4 km W of Muráň village, middle part of Hrdzavá dolina valley, 1063 m a.s.1., GPS = N48 44'00.978", E1959'00.947".

87 Muránska planina Mts, Muráň, $\mathrm{N}$ edge of Murán̆ village, $413 \mathrm{~m}$ a.s.l., GPS $=$ N48 $44^{\prime} 00.521^{\prime \prime}$, E2002'00.753".

88 Veporské vrchy Mts, Tisovec, $3.3 \mathrm{~km} \mathrm{NE}$ of Bánovo settlement, $2 \mathrm{~km}$ NE of Magnet hill, slope of Strieborná hill, 740 m a.s.1., GPS = N4843'59.100", E1953'42.300".

89 Muránska planina Mts, Tisovec, $5.6 \mathrm{~km} \mathrm{NW}$ of Tisovec town, $5.5 \mathrm{~km} \mathrm{NE}$ of Hradová hill, along Rimavá stream, $650 \mathrm{~m}$ a.s.1., GPS = N48 $43^{\prime} 58.501^{\prime \prime}$, E19 ${ }^{\circ} 56^{\prime} 00.121^{\prime \prime}$.

90 Stolické vrchy Mts, Muráň, 900 m NW of Muránska Lehota village, SW edge of Muráň village, 435 m a.s.l., GPS = N48 43'58.400", E2002'19.500".

91 Stolické vrchy Mts, Muránska Lehota, $250 \mathrm{~m}$ NW of Muránska Lehota village, $550 \mathrm{~m} \mathrm{~S}$ of Muráň village, loc. 'Dolný rybník' pond, 450 m a.s.1., GPS = N4843'57.500", E2002'49.200".

92 Veporské vrchy Mts, Tisovec, $3.2 \mathrm{~km}$ NE of Bánovo settlement, $1.9 \mathrm{~km} \mathrm{NE}$ of Magnet hill, slope of Strieborná hill, $770 \mathrm{~m}$ a.s.1., GPS = N4843'54.500", E1953'40.300".

93 Stolické vrchy Mts, Murán̆, $800 \mathrm{~m}$ SE of Muráň village, along a public road, $365 \mathrm{~m}$ a.s.l., GPS = N4843'55.600", E2003'58.300".

94 Muránska planina Mts, Muráň, 2 km NW of Muránska Lehota village, $1.8 \mathrm{~km}$ SW of Muráň village, loc. 'Javorníková' NNR, 674 m a.s.1., GPS = N4843'50.509", E2001'01.646".

95 Stolické vrchy Mts, Muránska Lehota, $150 \mathrm{~m} \mathrm{~W}$ of Muránska Lehota village, $900 \mathrm{~m}$ S of Murán̆ village, near loc. 'Dolný rybník' pond, 390 m a.s.1., GPS = N4843'49.400", E2002'45.300".

96 Muránska planina Mts, Tisovec, $5.1 \mathrm{~km} \mathrm{NW}$ of Tisovec town, $5.2 \mathrm{~km} \mathrm{NE}$ of Hradová hill, along Rimavá stream, 640 m a.s.1., GPS = N4843'42.240", E1955'50.099".

97 Muránska planina Mts, Muráň, $3 \mathrm{~km} \mathrm{~W}$ of Muránska Lehota village, $3.4 \mathrm{~km} \mathrm{SW}$ of Murán̆ village, loc. 'Paseky', 756 m a.s.1., GPS = N48 43'32.016", E2000'08.136".

98 Veporské vrchy Mts, Tisovec, $4.9 \mathrm{~km}$ NW of Tisovec town, $2.3 \mathrm{~km}$ NE of Magnet hill, slope of Huta hill, 805 m a.s.1., GPS $=$ N48 43'27.120", E1955'07.644".

99 Veporské vrchy Mts, Tisovec, 4 km NW of Tisovec town, 1.4 km SW of Hajnáš hill, loc. 'Kozák', 610 m a.s.1., GPS = N48 43'05.592", E1955'43.356".

100 Muránska planina Mts, Tisovec, $4.1 \mathrm{~km} \mathrm{NW}$ of Tisovec town, 4 km N of Hradová hill, along Rimavá stream, $570 \mathrm{~m}$ a.s.1., GPS $=$ N48 $43^{\prime} 03.781^{\prime \prime}, \mathrm{E} 19^{\circ} 55^{\prime} 32.819^{\prime \prime}$.

101 Muránska planina Mts, Muráň, 5 km SW of Muráň village, Martinova valley, loc. 'Šarkanica' NNR, 620 m a.s.1., GPS = N48 42'42.516", E1959'03.732".

102 Muránska planina Mts, Murán̆, $2.7 \mathrm{~km}$ NE of Tisovec village, $1.9 \mathrm{~km} \mathrm{NW}$ of Martinova dolina valley, slope of Šajba Mt., loc. 'Šarkanica' NNR, 910 m a.s.1., GPS = N4842'35.716", E1958'10.168".

103 Muránska planina Mts, Tisovec, $3.3 \mathrm{~km} \mathrm{NW}$ of Tisovec town, $3 \mathrm{~km} \mathrm{~N}$ of Hradová hill, along Rimavá stream, $510 \mathrm{~m}$ a.s.1., GPS $=$ N48 $42^{\prime} 27.900^{\prime \prime}, \mathrm{E} 19^{\circ} 55^{\prime} 13.501^{\prime \prime}$.

104 Stolické vrchy Mts, Tisovec, $3.2 \mathrm{~km}$ NE of Tisovec village, $5 \mathrm{~km} \mathrm{SW}$ of Muráň village, $583 \mathrm{~m}$ a.s.1., GPS = N48 42'00.555", E1959'00.782".

105 Muránska planina Mts, Tisovec, $1.8 \mathrm{~km}$ SE of Bánovo settlement, $668 \mathrm{~m}$ a.s.1., GPS = N4842'00.534", E1952'00.319".

106 Stolické vrchy Mts, Tisovec, $5.2 \mathrm{~km} \mathrm{SW}$ of Muránska Lehota village, $3.8 \mathrm{~km}$ NE of Tisovec village, foot of Šajba hill, 850 m a.s.1., GPS = N4841'30.768", E1959'49.236".

107 Muránska planina Mts, Tisovec, 0.9 km NW of Tisovec village - part Št’avica, near loc. 'Košianovo', 600 m a.s.1., GPS = N48 41'30.264", E1957'08.748".

108 Muránska planina Mts, Tisovec, 4.3 km SE of Bánovo settlement, $2.7 \mathrm{~km} \mathrm{NW}$ of Tisovec town, near loc. 'Suché doly' NR, 'Teplica', 490 m a.s.1., GPS = N4841'25.004", E1953'59.000".

109 Muránska planina Mts, Tisovec, $3 \mathrm{~km}$ NE of Tisovec village, $751 \mathrm{~m}$ a.s.1., GPS = N48 41'00.981", E1959'00.010".

110 Muránska planina Mts, Tisovec, $3.6 \mathrm{~km} \mathrm{~W}$ of Tisovec town, $2.5 \mathrm{~km} \mathrm{SW}$ of Hradová village, loc. 'Suché doly' NR, $625 \mathrm{~m}$ a.s.1., GPS $=$ N48 40'23.664", E1953'33.072".

111 Stolické vrchy Mts, Tisovec, $3.5 \mathrm{~km}$ NE of Rimavska Píla village, $3.3 \mathrm{~km}$ E of Tisovec town, loc. 'Trstie' NR, 1100 m a.s.1., GPS = N48³9'40.968", E1959'22.812".

Abbreviations: $\mathrm{NNR}=$ National Nature Reserve; $\mathrm{NR}=$ Nature reserve 


\section{A LIST OF SPECIES AND COLLECTION SITES OF RECENTLY RECORDED IN THE MURÁNSKA PLANINA NATIONAL PARK}

\section{Nyholmiella gymnostoma [CR], Fig 4}

Loc: 36,39 .

Rediscovered in Slovakia after 54 years.

\section{Nyholmiella obtusifolia}

Loc: $2,5,23,30,37,39,40,41,44,46,47,50,51,52,58,62,67,68,72,74,83,87,91,92,93,95,97,98,99$, $101,104,105,106,108,109,110,111$.

\section{Dorcadion affine}

Loc: $1,2,3,5,6,7,8,10,11,13,16,18,24,25,27,28,33,34,36,40,41,43,44,45,47,49,50,51,52,57,58$, $59,60,61,62,63,68,69,72,74,75,77,78,80,81,83,84,85,87,89,90,92,93,95,97,98,99,100,101,102$, $103,104,106,108,109,110,111$.

\section{Dorcadion affine var. bohemicum}

Loc: 25, 58, 63, 66, 68, 93, 102.

New taxon for Muránska planina National Park and the territory of Slovakia.

\section{Dorcadion rupestre}

Loc: 24 .

New species for Muránska planina National Park.

\section{[NT]}

Loc: $1,2,3,4,5,6,7,8,9,10,11,12,13,14,18,20,21,22,24,25,26,27,28,29,30,33,35,37,38,39,40,41$, $42,43,44,45,46,47,48,50,52,53,55,56,57,58,59,60,62,63,66,67,68,69,70,71,72,74,75,77,80,81$, $82,83,84,85,86,87,89,90,92,93,96,97,98,99,100,101,102,104,105,108,109,110,111$.

\section{Dorcadion striatum [VU], Fig 3}

Loc: $2,4,6,7,8,10,11,18,20,22,24,25,27,28,29,30,32,33,34,35,36,37,38,39,40,41,42,43,44,45,46$, $47,48,49,50,51,52,55,56,57,58,59,60,62,63,64,70,72,73,74,75,77,78,79,80,81,82,83,84,85,86$, $87,92,93,94,95,97,98,99,100,101,102,103,104,108,109,111$.

\section{Orthotrichum anomalum}

Loc: 2, 24, 35, 39, 42, 97, 98, 108.

\section{Orthotrichum cupulatum}

Loc: 35 .

\section{Orthotrichum diaphanum}

Loc: 26, 29, 35, 39, 47, 82, 83, 87, 93, 94, 97, 110.

New species for Muránska planina National Park.

\section{Orthotrichum pallens [NT], Fig 1}

Loc: $1,2,3,4,5,6,7,8,9,10,12,16,20,21,22,24,25,26,28,30,32,35,36,38,39,40,41,43,44,45,46,47$, $48,49,50,51,52,54,55,58,59,60,61,62,63,64,65,67,68,69,70,72,73,74,75,76,77,78,80,81,82,83$, $84,85,86,87,89,90,91,92,93,95,96,97,98,99,100,101,102,103,104,105,107,108,109,110,111$. 


\section{Orthotrichum patens [CR], Fig 2}

Loc: $2,8,10,20,25,33,34,35,36,39,41,42,43,45,47,48,51,55,58,60,61,62,63,64,65,69,71,72,73,79$, $80,81,82,83,84,85,88,90,92,93,97,98,99,101,102,103,104,105,106,107,108,109,110$.

New species for Muránska planina National Park.

\section{Orthotrichum pumilum}

Loc: $1,2,5,8,10,12,21,29,30,35,36,38,39,40,41,42,43,44,46,47,50,51,56,58,61,62,63,64,67,68$, 71, 72, 74, 75, 77, 78, 79, 83, 87, 91, 92, 93, 94, 95, 97, 98, 99, 102, 104, 110, 111.

\section{Orthotrichum rogeri [EX]}

Loc: 99.

Rediscovered in Slovakia after 77 years.

\section{Orthotrichum scanicum [CR]}

Loc: $57,82$.

New species for Muránska planina National Park.

\section{Orthotrichum stramineum [DD]}

Loc: $2,3,6,7,8,10,11,13,14,20,22,25,26,27,28,29,30,32,33,34,35,36,37,38,39,40,43,44,45,46,47$, $48,51,52,53,56,57,59,60,61,62,63,67,68,70,72,73,74,75,77,79,80,81,82,83,84,85,86,87,89,92$, 93, 96, 97, 98, 99, 100, 101, 102, 103, 105, 107, 108, 109, 110, 111.

New species for Muránska planina National Park

\section{Pulvigera lyellii [NT], Fig 5}

Loc: 22, 38, 52, 94, 101, 102, 107, 109.

New species for Muránska planina National Park

\section{Ulota bruchii}

[VU]

Loc: $3,7,8,10,12,17,19,25,28,29,31,32,33,34,35,39,41,45,47,48,53,54,59,63,65,70,71,80,81,82$, 86, 87, 92, 96, 97, 98, 99, 100, 101, 102, 103, 108, 109.

New species for Muránska planina National Park.

\section{[NT]}

Loc: $2,5,6,7,9,11,13,14,15,16,17,18,19,20,24,25,28,29,30,32,33,34,41,47,50,52,56,59,60,63,65$, $70,73,81,86,87,92,97,98,99,100,101,102,103,105,108,109$.

\section{Discussion}

As a result of the identification of approximately 500 specimens, 19 moss taxa belonging to 5 genera have been found in the study area. Among these mosses 8 taxa, namely Dorcadion affine var. bohemicum, D. rupestre, Orthotrichum diaphanum, O. patens, O. scanicum, O. stramineum, Pulvigera lyellii, and Ulota bruchii were recorded as a new for territory of the Muránska planina National Park and Dorcadion affine var. bohemicum is a new record for Slovakia. Eleven species are listed on the Red list of bryophytes of Slovakia (cf. Kubinská et al. 2001) - EX: Orthotrichum rogeri; CR: Nyholmiella gymnostoma, Orthotrichum patens, $O$. scanicum; VU: Dorcadion striatum, Ulota bruchii; NT: Dorcadion speciosum, Orthotrichum pallens, Pulvigera lyellii, Ulota crispa; DD: Orthotrichum stramineum.

Orthotrichum rogeri is listed also among the protected species in Slovakia (as defined in the Decree of the Ministry of Environment of the Slovak Republic No. 579 of 10 December 2008 Coll.). 

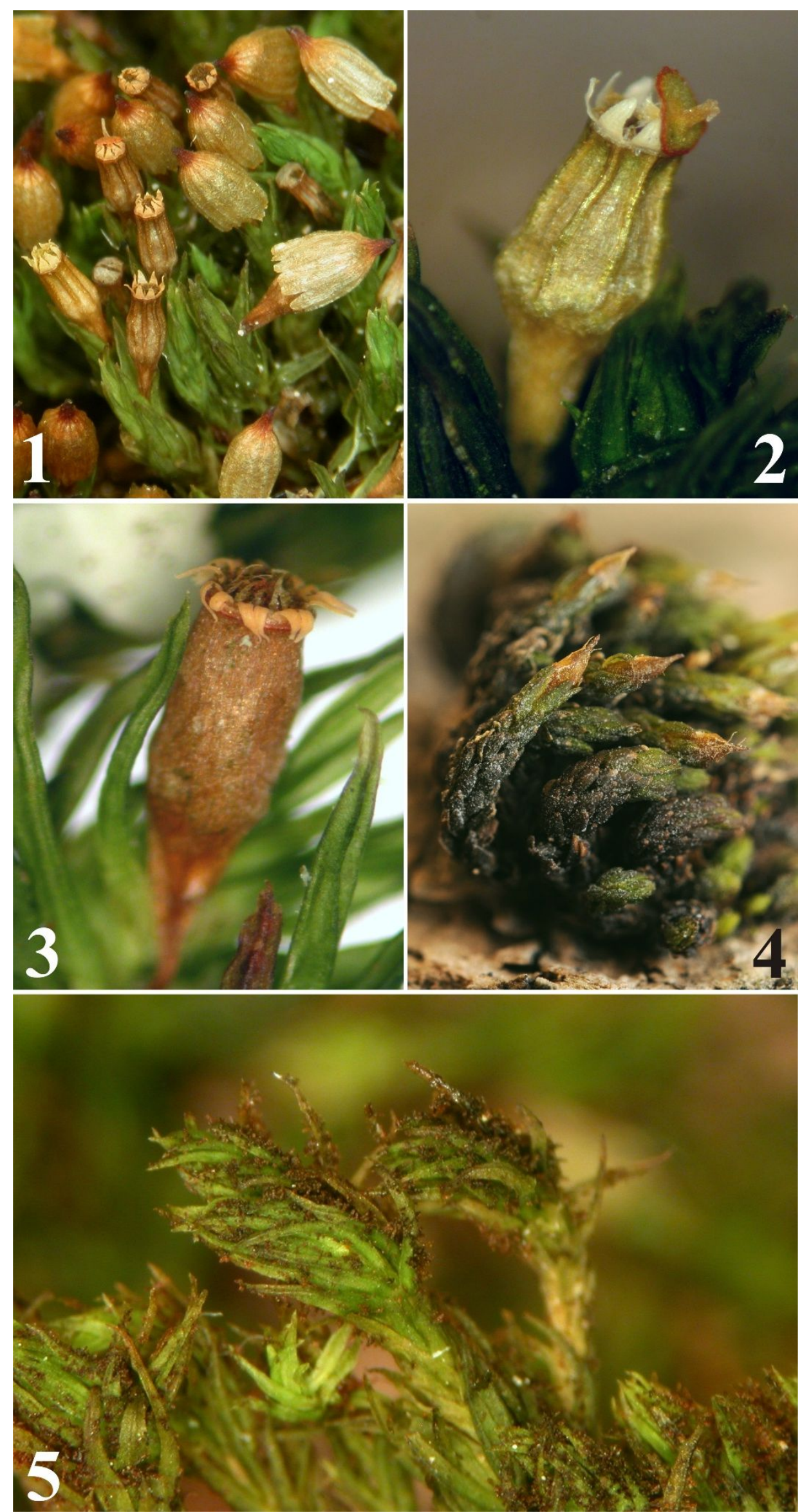

Figs 1-5: Noteworthy species recently recorded in Muránska planina: $\mathbf{1}-$ Orthotrichum pallens, $\mathbf{2}-$ O. patens, 3 -Dorcadion striatum, $\mathbf{4}$ - Nyholmiella gymnostoma, $\mathbf{5}$ - Pulvigera lyellii. Photos made by V. Plášek. 


\section{Dorcadion affine var. bohemicum}

The variety was described only recently by Plášek et al. (2011) from the Czech Republic, but now it appears this taxon is a geographically widespread. During 5 years it has already been reported from the USA (Ellis et al., 2012a), Poland (Ellis et al., 2012b), Sweden (Ellis et al., 2013), Ukraine (Ellis et al., 2014a), Belgium (Ellis et al., 2014b). The recent finding of this taxon in Muránska planina National Park is the first known record in Slovakia (cf. Ellis et al. 2016).

Dorcadion affine var. bohemicum was found in the study area at 7 localities situated between ca. 500 and $830 \mathrm{~m}$ a.s.1. Mostly it grows on bark of Fraxinus excelsior and Acer pseudoplatanus. All recorded populations were observed with large numbers of sporophytes.

\section{Dorcadion striatum}

The species is a common Dorcadion recorded in Muránska planina National Park. It was collected at 77 of 111 studied sites. It has been reported here already by Peciar (1974) but no details about its occurrence were published.

\section{Nyholmiella gymnostoma}

The species was collected in Slovakia for the first time in 1956 by Pilous in Muránska Huta village (Vondráček 1993). Subsequently, Pilous in 1957 (Pilous 1961) and Pospíšil in 1963 (Vondráček 1993) confirmed its occurrence in the locality. Muránska Huta village and its vicinity are situated in the buffer zone of Muránska planina National Park near the NE edge of the protected area. The occurrence of the species in its historical locality was confirmed after 54 years in 2015 and one new location was found there, on Šiance Mt. in 2016.

\section{Orthotrichum pallens}

The species is one of most common Orthotrichum recently recorded in Muránska planina National Park. It was collected at 87 of 111 studied sites.

\section{Orthotrichum patens}

In the last decade it seems that Orthotrichum patens is expanding its area in Central Europe as a consequence of improved air quality and possibly also as a result of climatic change (cf. Plášek 2012). Its present distribution in Slovakia is still, however, insufficiently known. In the area of Muránska planina it has not recorded before, but during the recent field survey it was located at 53 sites where it was found growing on the bark of a large spectrum of phorophytes, but mainly on Fagus sylvatica, Carpinus betulus or Acer pseudoplatanus.

\section{Orthotrichum scanicum}

In Slovakia the species was found for the first time by Podpera in 1922 in the High Tatra Mts. The second finding and last for a long time was collected by Pospíšil in 1977 in Vtáčnik Mts (Vondráček 1993). The recent survey in Muránska planina National Park brings new data about its occurrence in the area and confirms its presence in Slovakia. Orthotrichum scanicum was found at two sites situated near Fabova hol'a Mt. - on Kučelach Mt. and Kl'ak Mt. in the NW part of the National Park. It was recorded growing in small populations (in both of cases the size had no more than 2-3 $\mathrm{cm}^{2}$ ) on the bark of Fagus sylvatica and Coryllus avellana together with other epiphytic mosses, e.g. Dorcadion affine, D. speciosum, D. striatum, and Orthotrichum stramineum. 


\section{Orthotrichum rogeri}

From historical herbarium specimens and literature data, Orthotrichum rogeri was previously recorded only once in Slovakia. In 1938 J. Šmarda collected bryological material in Klatná valley ( $\mathrm{N}$ part of Muránska planina National Park) and identified it as Orthotrichum tenellum (original description of the locality is: valley of Klatná, bark of Fagus, $800 \mathrm{~m}$ a.s.1.; leg. J. Šmarda 11.8.1938). The specimen which is housed in BRNM (\#363329) was subsequently re-identified as $O$. rogeri (Vondráček 1993). The identity was confirmed by Vítězslav Plášek who had the opportunity to study the specimen in 2016.

In April 2015 the valley of Klatná was visited to confirm the presence of the species at this location. However, despite considerable sampling effort, the occurrence of the species could not be confirmed there. Nevertheless, Orthotrichum rogeri was later found in loc. Kozák, situated $c a .4 \mathrm{~km} \mathrm{NW}$ of Tisovec town. Three small metapopulations were recorded there growing on the bark of Fagus sylvatica together with O. pallens, Ulota bruchii and U. crispa. This finding of $O$. rogeri is confirmation of its occurrence in Slovakia after 77 years.

\section{Pulvigera lyellii}

Although formerly the species was placed in the genus Orthotrichum, its position there was no longer sustainable mainly due to its dioicous sexuality and plane leaf margins (cf. Plášek et al. 2015). Despite the fact that the plants of Pulvigera lyellii are usually sterile and do not produce capsules, they are easily recognized by the quantity of brownish gemmae situated over the leaf surfaces gives the impression that the leaves are dirty-dusty.

Until the present survey the species was not known from area of Muránska planina National Park. In 2015 it was found at 8 sites. It grows mostly on the bark of old trunks of Tilia cordata., T. platyphyllos, Fraxinus excelsior, and very rarely on Quercus pubescens between ca. 500900 m a.s.l.

Acknowledgements: We are very grateful to Dr. Rod Seppelt (Tasmania) for linguistic corrections of the manuscript. The contribution by V. Plášek and Zuzana Skoupá is part of a research project of the Institute of Environmental Techno-logies, reg. no. CZ.1.05/2.1.00/03.0100, Project LO1208 of the National Feasibility Programme I of the Czech Republic.

\section{References}

Blanár D. \& Kochjarová J. (2012): Flóra a vegetácia. [Národný park Muránska planina]. Krásy Slovenska 89(5-6): 22-25.

Ellis L.T., Alegro A., Bednarek-Ochyra H., Ochyra R., Bergamini A., Cogoni A., Erzberger P., Górski P., Gremmen N., Hespanhol H., Vieira C., Kurbatova L.E., Lebouvier M., Martinčič A., Asthana A.K., Gupta R., Nath V., Natcheva R., Ganeva A., Özdemir T., Batan N., Plášek V., Porley R.D., Randić M., Sawicki J., Schroder W., Sérgio C., Smith V.R., Sollman P., Ştefănuţ S., Stevens on C.R., Suárez G.M., Surina B., Uyar G. \& Surina Z.M. (2012a): New national and regional bryophyte records, 31. - Journal of Bryology 34(2): 123-134.

Ellis L.T., Bednarek-Ochyra H., Ochyra R., Cykowska B., Dulin M.V., Ezer T., Kara R., Flores J.R., Suárez G.M., Garcia C., Martins A., Sérgio C., Garilleti R., Kirmaci M., Agcagil E., Kurbatova L.E., Lebouvier M., Papp B., Szurdoki E., Philippov D.A., Plášek V., Pócs T., Sabovljević M., Sawicki J., Sim-Sim M., Szűcs P., Bidló A., Váňa J., Vigalondo B., Lara F., Draper I., Virchenko V.M. \& Wolski G.J. (2012b): New national and regional bryophyte records, 33. - Journal of Bryology 34(4): 281-291.

Ellis L.T., Bednarek-Ochyra H., Ochyra R., Benjumea M.J., Saïs L.V., Caparrós R., Lara F., Mazimpaka V., Dulin M.V., Garilleti R., Gremmen N., Grundling P.-L., Heras P., Infante M., Huttunen S., Ignatov M.S., Korvenpää T., Lebouvier M., Lewis Smith R.I., Lin S.-H., Yang J.-D., Linström A., Plášek V., Rosselló J.A., Sawicki J., van Rooy J. \& Smith V.R. (2013): New national and regional bryophyte records, 35. - Journal of Bryology 35(2): 129-139. 
Ellis L.T., Aleffi M., Asthana A.K., Srivastava A., Bakalin V.A., Batan N., Özdemir T., BednarekOchyra H., Borovichev E.A., Brugués M., Cano M.J., Choi S.S., De Beer D., Eckstein J., Erzberger P., Fedosov V.E., Ganeva A., Natcheva R., Garcia C.A., Sérgio C., Garilleti R., Albertos B., Puche F., Gücel S., Higuchi M., Hugonnot V., Hylander K., Kırmac1 M., Aslan G., Koponen T., Lara F., Mazimpaka V., van Melick H., Müller F., Özenoglu Kiremit H., Papp B., Szurdoki E., Plášek V., Č́ihal L., van der Pluijm A., Poponessi S., Mariotti M.G., Reyniers J., Sabovljević M.S., Sawicki J., Smith V.R., Stebel A., Ştefănuţ S., Sun B.-Y., Váňa J. \& Venanzoni R. (2014a): New national and regional bryophyte records, 40. - Journal of Bryology, 36(3): 223-244.

Ellis L.T., Aleffi M., Tacchi R., Alegro A., Alonso M., Asthana A.K., Sahu V., Biasuso A.B., Callaghan D.A., Ezer T., Kara R., Garilleti R., Gil-López M.J., Gwynne-Evans D., Hedderson T.A., Kiebacher T., Larrain J., Long D., Lüth M., Malcolm B., Mamontov Y.S., Newsham K.K., Nobis M., Nowak A., Ochyra R., Pawlikowski P., Plášek V., Č́lhal L., Potemkin A.D., Puche F., Rios D., Gallego M.T., Guerra J., Sawicki J., Schäfer-Verwimp A., Segarra-Moragues J.G., Šegota V., Sofronova E.V., Ştefănuţ S., Szücs P., Bidló A., Papp B., Szurdoki E., Tan B.C., Váňa J., Vigalondo B., Draper I., Lara F., Yoon Y.-J., Sun B.-Y. \& Nishimura N. (2014b): New national and regional bryophyte records, 41. - Journal of Bryology, 36(4): 306-324.

Ellis L.T., Asthana A.K., Srivastava P., Omar I., Rawat K.K., Sahu V., Cano M.J., Costa D.P., Dias E.M., Dias dos Santos N., Silva J.B., Fedosov V.E., Kozhin M.N., Ignatova E.A., Germano S.R., Golovina E.O., Gremmen N.J.M., Ion R., Ştefănuţ S., von Konrat M., Jimenez M.S., Suárez G.M., Kiebacher T., Lebouvier M., Long D.G., Maity D., Ochyra R., Parnikoza I., Plášek V., Fialová L., Skoupá Z., Poponessi S., Aleffi M., Sabovljević M.S., Sabovljević A.D., Saha P., Aziz M.N., Sawicki J., Suleiman M., Sun B.-Y., Váňa J., Wójcik T., Yoon Y.-J., Żarnowiec J. \& Larraín J. (2016): New national and regional bryophyte records, 46. - Journal of Bryology, 38(1): 47-63.

Kochjarová J., Škodová I. \& Blanár D. (2015): Thermophilous and mountain non-forest vegetation pattern on the crossing of Carpathian and Pannonian region: an example from Muránska planina Mts (Central Slovakia). - Tuexenia 35: 195-220.

Kubinská A., Janovicová K. \& Šoltés R. (2001): Aktualizovaný zoznam pečeňovek, rožtekov a machov Slovenska. - Bryonora 28: 4-10.

Mitter P. (1991): Povrch. - In: Vološcuk I. \& Pelikán V. (eds), Muránska planina chránená krajinná oblast'. Obzor, Bratislava, 18-27 pp.

Peciar V. (1974): Studia bryofloristica Slovaciae VI. - Acta Fac. Rerum Natur. Univ. Comenianae 22: 93-101.

Pilous Z. (1961): Fragmenta bryologica 41-50. - Preslia 33: 277-290.

Plášek V. (2012): Klíč pro determinaci zástupců rodů Orthotrichum a Nyholmiella v České republice. - Bryonora 50: 17-33.

Plášek V., Sawicki J., Marková I. \& Wierzcholska S. (2011): Orthotrichum affine var. bohemicum (Orthotrichaceae), a new variety of epiphytic moss from the Czech Republic. - Acta Societatis Botanicorum Poloniae 80(4): 335-340.

Plášek V., Sawicki J., Ochyra R., Szczecińska M. \& Kulik T. (2015): New taxonomical arrangement of the traditionally conceived genera Orthotrichum and Ulota (Orthotrichaceae, Bryophyta). Acta Musei Silesiae 64: 169-174.

Vondráček M. (1993): Revize a rozšíření druhů rodu Orthotrichum Hedw. v České a Slovenské republice (Musci). - Sborník Západočeského Muzea v Plzni 85: 1-76.

Vyhláška Ministerstva životného prostredia Slovenskej republiky č. 579 z 10. decembra 2008, ktorou sa mení vyhláška Ministerstva životného prostredia Slovenskej republiky č. 24/2003 Z. z., ktorou sa vykonáva zákon č. 543/2002 Z. z. o ochrane prírody a krajiny v znení neskorších predpisov [Decree of the Ministry of Environment of the Slovak Republic No. 579 of 10 December 2008 amending the Decree of the Ministry of Environment of the Slovak Republic No. 24/2003 Coll., implementing the Act No. 543/2002 Coll. on Nature and Landscape Protection as later amended].

Authors' addresses: Vítězslav Plášek, Lucie Fialová \& Zuzana Skoupá, Dept. of Biology and Ecology, University of Ostrava, Chittussiho 10, CZ - 74601 Ostrava, Czech Republic.

Corresponding author: E-mail: vitezslav.plasek@osu.cz

Drahoš Blanár, Administration of the Muránska planina National Park, J. Král'a 12,

SK - 05001 Revúca, Slovakia.

E-mail: drahos.blanar@sopsr.sk 